\title{
LA CREACIÓN DE EMPRESAS POR GRADUADOS/AS DE LA UNIVERSIDAD ESTATAL A DISTANCIA DE COSTA RICA*
}

\author{
ROSBERLY ROJAS CAMPOS \\ Centro de Investigación y Evaluación Institucional \\ Universidad Estatal a Distancia, Costa Rica. \\ rrojas@uned.ac.cr
}

\section{RESUMEN}

En 2008, se aplicó un cuestionario a 19 graduados/as de diferentes disciplinas de la Universidad Estatal a Distancia (UNED), que habian creado sus propios negocios, para conocer su experiencia durante la creación y primeros años de la empresa. Los resultados muestran que la mayoría había creado microempresas comerciales y de servicios; y se enfrentaron a problemas como una débil formación en temáticas de negocios, poca integración a redes y equipos de socios, e insuficiente apoyo de la universidad. Asimismo, tuvieron problemas de financiamiento, recurrieron a los ahorros propios y familiares con poco acceso al sistema financiero. Esto implicó la reducción de la escala del proyecto, el crecimiento de la empresa a una tasa menor a la deseada y la dedicación del emprendedor/a a otros proyectos adicionales que le generaran liquidez y capital. Durante los primeros años de funcionamiento, conseguir clientes y proveedores adecuados fueron dificultades importantes.

PALABRAS CLAVE: MICROEMPRESA, PEQUENA Y MEDIANA EMPRESA, EMPRENDEDURISMO, FORMACIÓN UNIVERSITARIA, MIPYMES.

* Para la realización de esta investigación se contó con el apoyo en recursos humanos y materiales del Centro de Investigación y Evaluación Institucional de la UNED.

\section{ABSTRACT}

In 2008, we interviewed 19 graduates of the Universidad Estatal a Distancia of Costa Rica who had started their own business, to learn about their experience during the first years of the company. The interviews were based on a questionnaire. Most had had created micro-enterprises in the Trade and Services sector, and had faced weak knowledge of business management, little integration to business networks and insufficient support from the University. Also, they did not have enough funds to be competitive in the market. They principally used own and family savings, and rarely access to the financial market. This implied the reduction of the scale of the project, in the growth rate of the company and the entrepreneur dedication to additional projects to get liquidity and capital. During the first years, get customers and appropriate suppliers were significant difficulties.

KEY WORDS: SMALL AND MEDIUM ENTERPRISE, ENTREPRENEURSHIP, UNIVERSITIES, SMES.

\section{INTRODUCCIÓN}

La presente investigación pretende contribuir al análisis de un sector fundamental en la economía nacional: las micro, pequeñas y medianas empresas (MIPYMES). Específicamente, se pretende estudiar la creación de MIPYMES por parte de las personas graduadas universitarias de la UNED.

Para ello se analizan los factores que estimulan o limitan ese proceso: la identificación de la oportunidad, la elaboración del proyecto, la decisión 
final, el acceso y movilización de los recursos necesarios, el ingreso al mercado y la gestión de la empresa durante los primeros años de vida. Además, se identifican algunos elementos relacionados con la contribución actual y potencial de la Universidad respecto del surgimiento de personas emprendedoras.

La relevancia de la temática de este estudio es creciente en América Latina. Cada vez más, las universidades muestran mayor compromiso con el desarrollo de las capacidades emprendedoras en su alumnado. Sin embargo, la investigación sobre las características de las empresas y del proceso emprendedor de las poblaciones universitarias en el contexto latinoamericano es aún incipiente, pero con un creciente interés en los últimos años (Kantis, 2004).

Dada la importancia que tienen las MIPYMES en la estructura productiva nacional, las universidades públicas costarricenses están fortaleciendo la vinculación con este sector. En este sentido, la UNED posee una ventaja competitiva importante, dada su característica de ofrecer educación a distancia, para llegar a una población amplia de estudiantes universitarios en todas las regiones del país con capacitación para fortalecer la capacidad emprendedora, utilizando las tecnologías de información y comunicación.

Se espera que los resultados presentados aquí puedan servir a las unidades académicas para enriquecer sus planes de estudio y coadyuvar con el fomento de los emprendimientos de sus estudiantes, como parte de la respuesta a la pertinencia social de la Universidad; dado que las evidencias muestran que las nuevas empresas fundadas por personas graduadas de las universidades están llamadas a jugar un rol importante como agentes de cambio. Este rol es crítico en la sociedad del conocimiento, especialmente en los países emergentes, como es el caso de Costa Rica, donde las empresas existentes no juegan el rol de organizaciones incubadoras para nuevos emprendimientos (Postigo \& Tamborín, 2004).
Además, en América Latina, el 50\% de las empresas calificadas como más dinámicas fueron creadas por personas graduadas de las universidades, y este porcentaje se incrementa a $66 \%$ si se consideran también a aquellos con nivel universitario incompleto; asimismo, las tasas de fracaso de nuevas empresas creadas por quienes se graduaron de la universidad eran menores al $20 \%$, mientras que las de personas con poca formación alcanzan hasta el 80\% (Kantis, Ishida \& Komori, 2002).

\section{METODOLOGÍA}

Para llevar a cabo la investigación se aplicó un cuestionario a 19 personas graduadas de la UNED que habían creado sus empresas en los últimos años, con el fin de obtener información respecto a las características de su empresa y el proceso que realizaron para formarla, con sus fortalezas y debilidades. Estas personas graduadas no recibieron capacitación formal en emprendedurismo durante su carrera universitaria en la UNED.

\section{Población}

La población estudiada corresponde a las personas graduadas de la UNED, en las carreras de grado, durante los años 2005, 2006, y la primera graduación de 2007, que habían creado su empresa y la mantenían vigente.

Dado que no se cuenta en la UNED con una base de datos de emprendedores, se usó la base de datos de graduados, que elabora el Centro de Investigación y Evaluación Institucional. Ésta contiene la información recolectada por medio de una boleta, que es administrada a cada estudiante en el momento en que se gradúa.

Se seleccionaron las 102 personas que, según la boleta, laboraban en empresa propia o familiar. A cada una de ellas se les consultó por teléfono (según el número reportado en la boleta) si tra- 
bajaban en una empresa de su propia creación. Un total de 27 personas habían creado su empresa y la mantenían vigente.

A las 27 personas se les contactó por teléfono, por segunda vez, para aplicarles un cuestionario. Se realizaron como máximo tres intentos por persona. De ellas, se logró obtener 19 cuestionarios llenos, ocho no respondieron, aduciendo principalmente falta de tiempo.

\section{Instrumento para la recolección de la información}

El cuestionario aplicado a las personas graduadas se construyó con base en el denominado "Estudio Comparado sobre Entrepreneurship en América Latina y Este de Asia" empleado en la investigación "Empresarialidad en economías emergentes: Creación y desarrollo de nuevas empresas en América Latina y el Este de Asia" (Kantis, Ishida \& Komori, 2002), debido a que éste es un instrumento validado al haber sido aplicado a 1220 personas creadoras de nuevas empresas en diferentes países de América Latina y Asia, incluyendo a Costa Rica.

En la categoría "La empresa" se consulta sobre las características principales de la empresa formada. En la categoría "La persona empresaria" se pregunta sobre la persona y sus motivaciones para emprender, las competencias y conocimientos con que contaban para realizar la actividad emprendedora y la experiencia anterior que sirvió de base para formar su negocio. En la categoría "El mercado y el contexto" se tratan los aspectos relacionados con la información para la toma de decisiones, y las dificultades y apoyos para la creación de las empresas (ver Tabla 1).

TABLA 1

\section{CATEGORÍAS Y VARIABLES DEL CUESTIONARIO UTILIZADO EN EL PRESENTE ESTUDIO}

\begin{tabular}{|c|c|c|}
\hline CATEGORÍA & VARIABLE & NÚMERO DE PREGUNTAS \\
\hline \multirow[t]{7}{*}{ La empresa } & Ubicación & 1 \\
\hline & Actividad principal & 1 \\
\hline & Tamaño de la empresa & 1 \\
\hline & Tipo de mercado & 2 \\
\hline & Relación con los competidores & 3 \\
\hline & Tipo de empresa & 2 \\
\hline & Planes a futuro & 2 \\
\hline \multirow[t]{7}{*}{ La persona empresaria } & Formación universitaria & 1 \\
\hline & Ocupación de la madre y el padre durante la niñez de las personas graduadas & 2 \\
\hline & Experiencia laboral & 2 \\
\hline & Motivaciones para ser empresario/a & 2 \\
\hline & Competencias y conocimientos en el momento de creación de la empresa & 2 \\
\hline & Experiencia en la creación de empresas: empresas fallidas & 4 \\
\hline & Asociatividad & 3 \\
\hline \multirow[t]{5}{*}{ El mercado y el contexto } & Información y fuentes sobre oportunidades de negocios & 2 \\
\hline & Información para la toma de decisiones & 1 \\
\hline & Dificultades para creación y puesta en marcha de la empresa & 3 \\
\hline & Financiamiento & 3 \\
\hline & Apoyo de la universidad & 3 \\
\hline
\end{tabular}

Fuente: Elaboración propia. 


\section{Análisis de la información}

El análisis de la información se realizó por medio de la descripción de la distribución de las variables, obteniéndose las tablas de frecuencias absolutas y relativas, y los gráficos de barras.

\section{RESULTADOS}

La situación de Costa Rica es similar a la de la mayoría de los países en desarrollo, en los que las MIPYMES son una fuente importante de empleo, pero no se desempeñan como agentes de innovación y promoción de la eficiencia económica (Ulate, 2001). En 2008, el parque empresarial privado costarricense dominado por la micro (67\%) y pequeña empresa (26\%), generaba el 32\% del empleo nacional (MEIC, 2009). Sin embargo, aunque un 76\% de las empresas exportadoras eran MIPYMES su participación relativa en el valor exportado alcanzó solamente el 10\% (Procomer, 2008).

No existe una definición mundial uniforme para clasificar las MIPYMES, ésta se realiza de acuerdo con el contexto económico de cada país y las características de su propia industria (Zevallos, 2003). En la mayoría de los países de Latinoamérica, el indicador principal es el empleo (Hernández, 2001; Ulate, 2001). En el caso de Costa Rica, el Ministerio de Economía, Industria y Comercio (MEIC), basado en el Reglamento a la Ley 8262, establece la diferenciación entre micro, pequeña o mediana empresa. Sin embargo, dada la complejidad de la fórmula, el citado Ministerio presenta la información estadística de las MIPYMES utilizando para su clasificación el número de personas empleadas, de la siguiente manera: microempresas (de 1 a 5), pequeñas (de 6 a 30), medianas (de 31 a 100), grandes (más de 100), clasificación que será usada en esta investigación.

En Centroamérica, desde los años 90, se han establecido una serie de políticas y programas para fortalecer las MIPYMES y convertirlas en factor de desarrollo. Este hecho se refleja en la gran cantidad de organizaciones que brindan apoyo a este sector, de tipo multilateral: Gesellschaft für Technische Zusammenarbeit (GTZ, por sus siglas en alemán), Banco Interamericano de DesarroIlo (BID), Organización de Naciones Unidas para el Desarrollo Industrial (ONUDI) Agencia para el Desarrollo Internacional (USAID, por sus siglas en inglés), Agencia de Cooperación Internacional de Japón (JICA, por sus siglas en inglés), entre otros; además de las 85 organizaciones de ámbito regional y nacional, de las cuales un 50\% se encuentran en Costa Rica (Fundación Arias para la Paz y el Progreso Humano, 2006).

Uno de los principales desafíos en materia económica para los países de Centroamérica es incorporar las MIPYMES en los sectores productivos exitosos tanto para el ámbito nacional como para el internacional. Hernández (2001) propone considerar el enfoque sistémico para que las MIPYMES contribuyan con mayor valor agregado al Producto Interno Bruto (PIB), es decir, un enfoque que considera los cuatro niveles determinantes del desempeño de las MIPYMES, a saber: el microeconómico (dentro de las empresas, para crear ventajas competitivas); el meso económico (eficiencia del entorno, mercados de factores, infraestructura física e institucional $y$, en general, las políticas específicas para la creación de ventajas competitivas); el macroeconómico (política económica) y, finalmente, el meta económico o estratégico (estructura política y económica orientada al desarrollo, estructura competitiva de la economía, visiones estratégicas, planes nacionales de desarrollo).

Según la Comisión Económica para América Latina (CEPAL, 1999) las dificultades de la pequeña y mediana empresa centroamericana se relacionan con su tamaño y poder de mercado, los recursos humanos, el aprovisionamiento de insumos, la gestión empresarial y la vulnerabilidad ante condiciones macroeconómicas. En cuanto al tamaño, se citan las dificultades para aprovechar las economías de escala y el reducido po- 
der de mercado, lo que limita su capacidad de negociación. El factor humano también es una limitante, ya que se hace difícil atraer y mantener personal de elevada calificación, por el nivel de los sueldos. En cuanto a las materias primas, CEPAL (1999) hace referencia a su escasez, su calidad irregular, la variabilidad de precios y los plazos de entrega. En relación con la gestión, se menciona la centralización de las funciones de administración y la gestión en la persona empresaria/a-dueño/a, lo cual crea una presión por resolver las necesidades de corto plazo de la empresa y descuidar la planificación de estrategias de largo plazo. Una limitante que menciona el estudio de la CEPAL (1999) es la alta vulnerabilidad a las fluctuaciones de la actividad económica, en comparación con las empresas de mayor tamaño que cuentan con mayores herramientas para paliar las crisis económicas.

En cuanto al tema de la gestión, de la revisión de publicaciones internacionales que tratan sobre emprendedurismo y empresarios, se puede decir que la capacidad emprendedora está afectada por factores personales y factores del entorno. Entre los factores relacionados con la personalidad, se tiene la creatividad, la alta capacidad para anticiparse a las oportunidades y a las acciones, y para la orientación de objetivos y la búsqueda de soluciones, la necesidad de realización, la fuerza de voluntad para tomar riesgos, el control interno, el espíritu independiente que permita la libertad, la autonomía y la habilidad para trabajar para uno mismo, la adaptabilidad y capacidad de aprender, la organización y el trabajo en equipo, la competitividad y la capacidad para delegar obligaciones y no responsabilidades. Entre los factores del entorno, se considera que los principales son la formación universitaria y la experiencia empresarial de la persona, tanto en su familia como en trabajos anteriores (Ribeiro, 2004).

\section{Los emprendimientos en micro, pequeña y mediana empresa de quienes se han graduado de la UNED}

A continuación se presentan los resultados obtenidos de la aplicación de la encuesta a las personas graduadas de la UNED, entre 2005 y el primer ciclo de 2007, que reportaron ser personas emprendedoras de sus propios negocios. Estos resultados se agrupan en tres categorías: la empresa, la persona empresaria, y el mercado y el contexto.

En la categoría Empresa se resumen las características principales de la empresa formada en relación con el tamaño, localización, actividad principal, la situación de la competencia y los planes a futuro.

En la segunda categoría, la persona empresaria, se resumen las motivaciones para emprender, las competencias y conocimientos y la experiencia anterior que sirvió de base para formar su negocio.

En la categoría del mercado y el contexto se analizan los aspectos relacionadas con la información para la toma de decisiones, las dificultades y apoyos para la creación de la empresa.

\section{La Empresa}

En relación con las características de las empresas que han formado las graduadas/os de la UNED en el periodo de estudio, se aglomeran en cuatro provincias del país, a saber San José (42\%), Alajuela (32\%), Cartago (16\%) y Heredia (10\%), principalmente en cantones del Gran Área Metropolitana. En su mayoría se dedican al comercio y los servicios (Tabla 2). 
TABLA 2

\section{SECTOR Y TIPO DE ACTIVIDAD DE LAS EMPRESAS}

\begin{tabular}{|l|c|c|}
\hline \multicolumn{1}{|c|}{ SECTOR/ TIPO DE ACTIVIDAD } & \multicolumn{2}{c|}{ NÚMERO DE EMPRESAS } \\
\hline Comercio y servicios & & 5 \\
\hline Servicios educativos & & 2 \\
\hline Servicios de alimentación & & 1 \\
\hline Servicios de alojamiento & & 1 \\
\hline Servicios de salud & & 1 \\
\hline Servicios de limpieza y mantenimiento & & 1 \\
\hline Servicios de atención personal & & 1 \\
\hline Comercio minorista & & 16 \\
\hline Comercio mayorista & & \\
\hline & Subtotal & 1 \\
\hline Industrial & & 1 \\
\hline Artesanías & & 1 \\
\hline & Subtotal & \\
\hline Agrícola & & 1 \\
\hline Floricultura & & \\
\hline Producción de leche & & \\
\hline & & \\
\hline Total & & \\
\hline
\end{tabular}

Fuente: Elaboración propia.

De las empresas estudiadas, solo una es de tamaño pequeño y las demás son microempresas. Una de las empresas comenzó siendo micro pero pasó a ser pequeña.

En su mayoría $(73,7 \%)$ son empresas jóvenes, con menos de 10 años de creadas (Tabla 3).

TABLA 3

\section{AÑOS DE FUNCIONAMIENTO DE LAS EMPRESAS}

\begin{tabular}{|l|c|c|}
\hline \multicolumn{1}{|c|}{ Años } & Porcentaje & $\begin{array}{c}\text { Porcentaje } \\
\text { acumulado }\end{array}$ \\
\hline Menos de 1 año & 10,5 & 10,5 \\
\hline De 1 a menos de 4 años & 15,8 & 26,3 \\
\hline De 4 a menos de 10 años & 47,4 & 73,7 \\
\hline Más de 10 años & 26,3 & 100,0 \\
\hline Total & 100,0 & \\
\hline
\end{tabular}

Fuente: Elaboración propia.
Además, son empresas que se formaron rápidamente. El 88,2\% de los empresarios/as decidieron formar la empresa cuando había pasado un año o menos de que habían tenido la idea del negocio. Asimismo, transcurrieron seis meses o menos desde la decisión de comenzar hasta que inició el negocio para $87.4 \%$ de los casos. En conjunto, la mitad de los empresarios/as empezó su empresa apenas ocho meses después de que habían tenido la idea del negocio.

Respecto al tipo de mercado al que se dirigen, en su mayoría (63\%) son empresas dedicadas a vender sus productos y servicios a clientes finales, solamente un $21 \%$ lo hace a otras empresas o negocios, y un 16\% divide sus ventas entre clientes finales final y otras empresas.

Las personas entrevistadas señalaron tres estrategias principales en la captación de mercado para sus productos o servicios. En su mayoría, aplican la estrategia de diferenciación de su producto respecto a las demás empresas de su sector. Otro grupo cataloga sus emprendimientos como innovadores, con productos o servicios dirigidos al mercado local. El tercer grupo basa su competitividad en cobrar precios bajos para ganar una mayor proporción de mercado (Tabla 4).

\section{TABLA 4 \\ OPINIÓN DE LAS PERSONAS ENTREVISTADAS SOBRE LA CARACTERÍSTICA INNOVADORA $O$ COMPETITIVA DE LA EMPRESA}

\begin{tabular}{l|c}
\multicolumn{1}{c|}{ Característica } & Porcentaje \\
\hline Competitiva con base a diferenciación producto & 47,1 \\
Innovadora para el mercado local & 29,4 \\
Competitivos con base en bajos precios & 23,5 \\
\hline Total & 100,0 \\
\hline
\end{tabular}

Fuente: Elaboración propia. 
Como parte de las estrategias de mercado, quienes fueron entrevistados mencionaron el servicio personalizado a los clientes, la mejora continua y la incorporación del componente ambiental en el producto ofrecido.

En relación con los competidores, las empresas valoran mejor su posición respecto a la competencia en cuanto a las características relacionadas con la calidad y los servicios que brindan a los clientes. Respecto a la competitividad en precios, el diseño del producto, el servicio de entrega, el financiamiento de la venta, el segmento socioeconómico de los clientes y el posicionamiento del producto, se consideran en una situación similar a la de la competencia.

En cuanto a la posición del producto respecto al de sus competidores, las personas entrevistadas aprecian ventajas en la calidad percibida $(64,7 \%)$, el diseño (60\%), la durabilidad (58,3\%), la fiabilidad $(57,1 \%)$ y el rendimiento $(53,3 \%)$. La funcionalidad del producto y los servicios asociados a éste se valoran en una posición similar con respecto a los productos de la competencia.

Se encontró mayor optimismo con respecto al producto que con respecto a la empresa, pues en relación con ésta solo se valoraron mejor que la competencia en dos de las ocho características, pero en relación con el producto se evaluaron mejor en cinco de las siete características.

Acerca de los planes a futuro, en el mediano plazo (cinco años) se observa la importancia que tiene generar mayor valor agregado a su producción, por medio de la inversión en la capacidad de producción, en el recurso humano y en la generación de conocimiento tecnológico aplicado a su producto (Figura 1).

\section{PLANES DE EXPANSIÓN DE LA EMPRESA EN LOS PRÓXIMOS CINCO AÑOS}

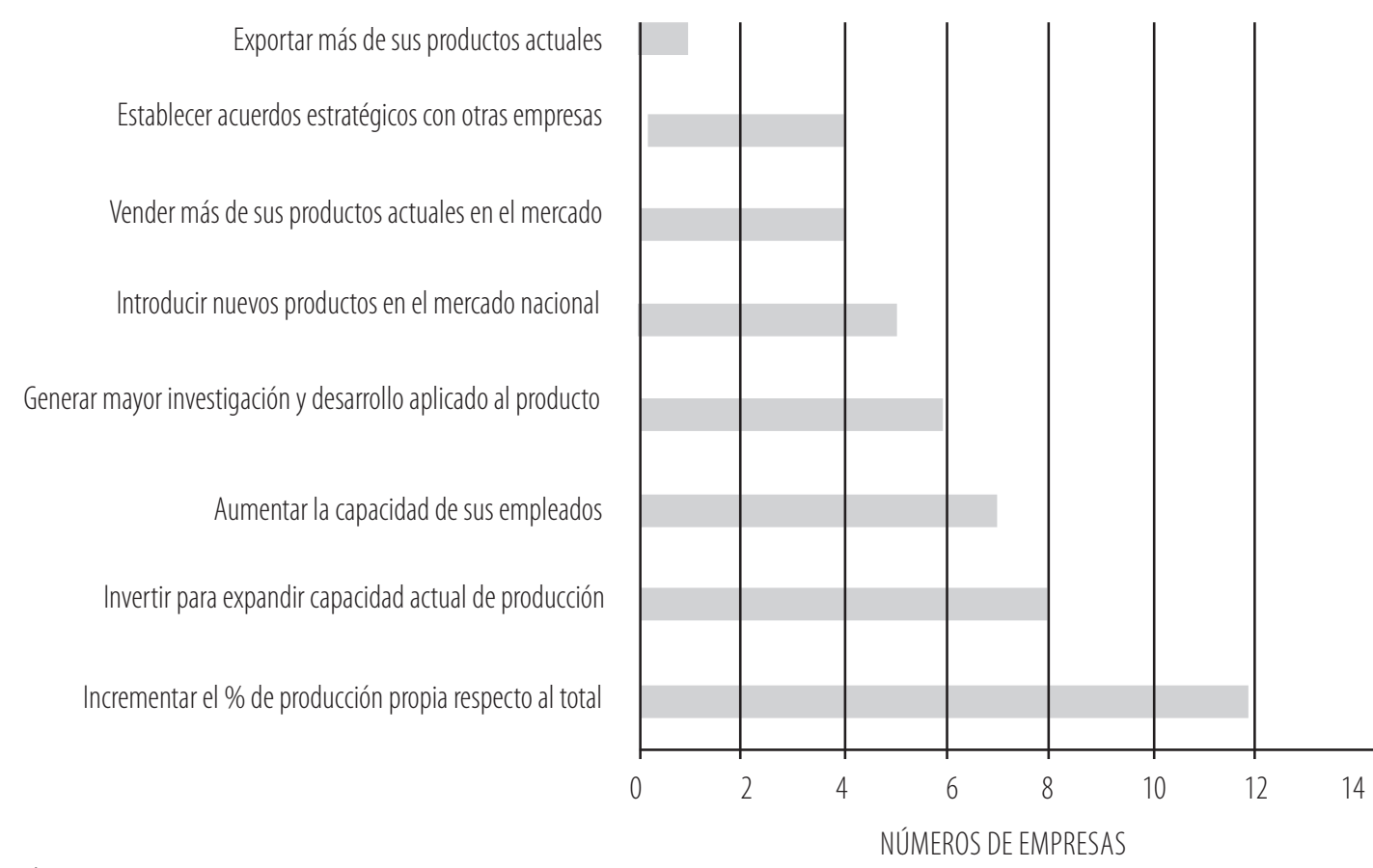

Fuente: Elaboración propia. 


\section{La persona empresaria}

En este apartado se presentan los resultados referentes a las características de las personas graduadas de la UNED que crearon sus propias empresas.

Las personas entrevistadas tienen formación en diferentes carreras de la UNED (Tabla 5). La mayoría $(83,3 \%)$ se encontraban satisfechas con la carrera en que se graduaron de la UNED y solamente un $16,7 \%$ parcialmente satisfechas.

TABLA 5

\section{TÍTULOS OBTENIDOS POR LOS ENTREVISTADOS PARA LAS DIFERENTES ESCUELAS DE LA UNED}

\begin{tabular}{|c|c|}
\hline $\begin{array}{l}\text { NÚMERO DE } \\
\text { PERSONAS }\end{array}$ & ESCUELA/TíTULO \\
\hline & Escuela de Ciencias de la Educación \\
\hline 2 & Bachillerato en Ciencias de la Educación I y II ciclos en Inglés \\
\hline 1 & Bachillerato en Educación Preescolar \\
\hline 2 & Diplomado en Educación General Básica l y II ciclos \\
\hline 1 & Diplomado en Educación Preescolar \\
\hline 1 & Licenciatura en Docencia \\
\hline 1 & Licenciatura en Educación Preescolar \\
\hline 8 & Subtotal \\
\hline & Escuela de Ciencias de la Administración \\
\hline 1 & Bachillerato Administración de Empresas \\
\hline 1 & Bachillerato Administración de Empresas énfasis en Contaduría \\
\hline 1 & $\begin{array}{l}\text { Bachillerato Administración de Empresas énfasis Gestión } \\
\text { Oraanizacional }\end{array}$ \\
\hline 2 & Licenciatura Administración de Empresas énfasis en Contaduría \\
\hline 1 & Licenciatura Administración de Empresas énfasis en Mercadeo \\
\hline 6 & Subtotal \\
\hline & Escuela de Ciencias Exactas y Naturales \\
\hline 1 & Bachillerato Enseñanza de la matemática \\
\hline 1 & Diplomado Administración de servicios de salud \\
\hline 1 & Licenciatura Administración de empresas Agropecuarias \\
\hline 1 & Licenciatura Manejo de recursos naturales \\
\hline 1 & Técnico Gestión Agroindustrial \\
\hline 5 & Subtotal \\
\hline 19 & Total \\
\hline
\end{tabular}

Fuente: Elaboración propia.
En relación con la edad, las personas emprendedoras representan los diferentes grupos: un $31,6 \%$ de 20 a 29 años, un $26.3 \%$ de 30 a 39 años y un $42,1 \%$ de 40 o más años. La mayoría son mujeres empresarias (73,7\%).

Respecto a la ocupación de sus padres durante la niñez de quienes fueron entrevistados, un $35,3 \%$ son hijos/as de padres empleados en el sector público y un 52,6\% de las madres se desempeñaban en el trabajo del hogar. Un porcentaje muy bajo tuvieron padres o madres empresarias (Tabla 6).

\section{TABLA 6}

\section{OCUPACIÓN DEL PADRE Y LA MADRE DURANTE LA NIÑEZ DE LA PERSONA ENTREVISTADA}

\begin{tabular}{lll}
\hline \multicolumn{1}{c}{ OCUPACIÓN } & \multicolumn{2}{c}{ PORCENTAJES } \\
\cline { 2 - 3 } & Padre & Madre \\
\hline Empleado/a del sector público & 35,3 & 21,1 \\
Trabajo no remunerado en el hogar & 0 & 52,6 \\
Empleado/a de empresa privada & 29,4 & 15,8 \\
Autoempleado/a (agricultor, comerciante, etc.) & 23,5 & 5,3 \\
Empresario/a & 5,9 & 5,3 \\
Profesión liberal & 5,9 & 0 \\
\hline Total & 100,0 & 100,0 \\
\hline
\end{tabular}

Fuente: Elaboración propia.

Un 67\% de las personas entrevistadas había ganado experiencia laboral antes de iniciar su propia empresa, de ellas un 41,7\% en PYMES y un $58,3 \%$ en empresas grandes. Un 41,7\% obtuvo la experiencia en un sector productivo semejante y un $58,3 \%$ en uno completamente diferente.

Al ser consultados sobre las motivaciones que le indujeron a ser emprendedor/a, las personas entrevistadas destacaron el deseo de trabajar en lo propio y así aprovechar los conocimientos adquiridos durante la formación profesional y la experiencia laboral en su propio negocio (Tabla 7). 
TABLA 7

\section{PRINCIPALES MOTIVACIONES PARA EMPRENDER}

\begin{tabular}{l|c}
\multicolumn{1}{c|}{ MOTIVACIONES } & PORCENTAJE \\
\hline Dejar de trabajar como empleado/a & 23,4 \\
Poner en práctica sus propias ideas y conocimientos & 21,3 \\
Lograr mayores ingresos económicos & 14,9 \\
Aprovechar las oportunidades que presentaba & 10,6 \\
el mercado & 10,6 \\
Alcanzar realización personal & 6,4 \\
Seguir el ejemplo de un empresariado exitoso & 6,4 \\
Contribuir al desarrollo económico del país & 4,3 \\
Emplearse pues estaba desempleado & 2,1 \\
\hline Lograr estatus y prestigio & 100 \\
\hline Total & \\
\hline
\end{tabular}

Fuente: Elaboración propia.
Sobre las competencias y conocimientos con que las personas entrevistadas contaban en el momento de creación de la empresa, fueron mencionadas principalmente aquellas relacionadas con la capacidad para relacionarse con los demás, negociar, crear, trabajar en equipo, solucionar problemas y la vocación por los negocios. La base de conocimientos sobre las temáticas de la administración de negocios, en la mayoría, era débil al iniciar la empresa, excepto en el caso de recursos humanos (Figura 2).

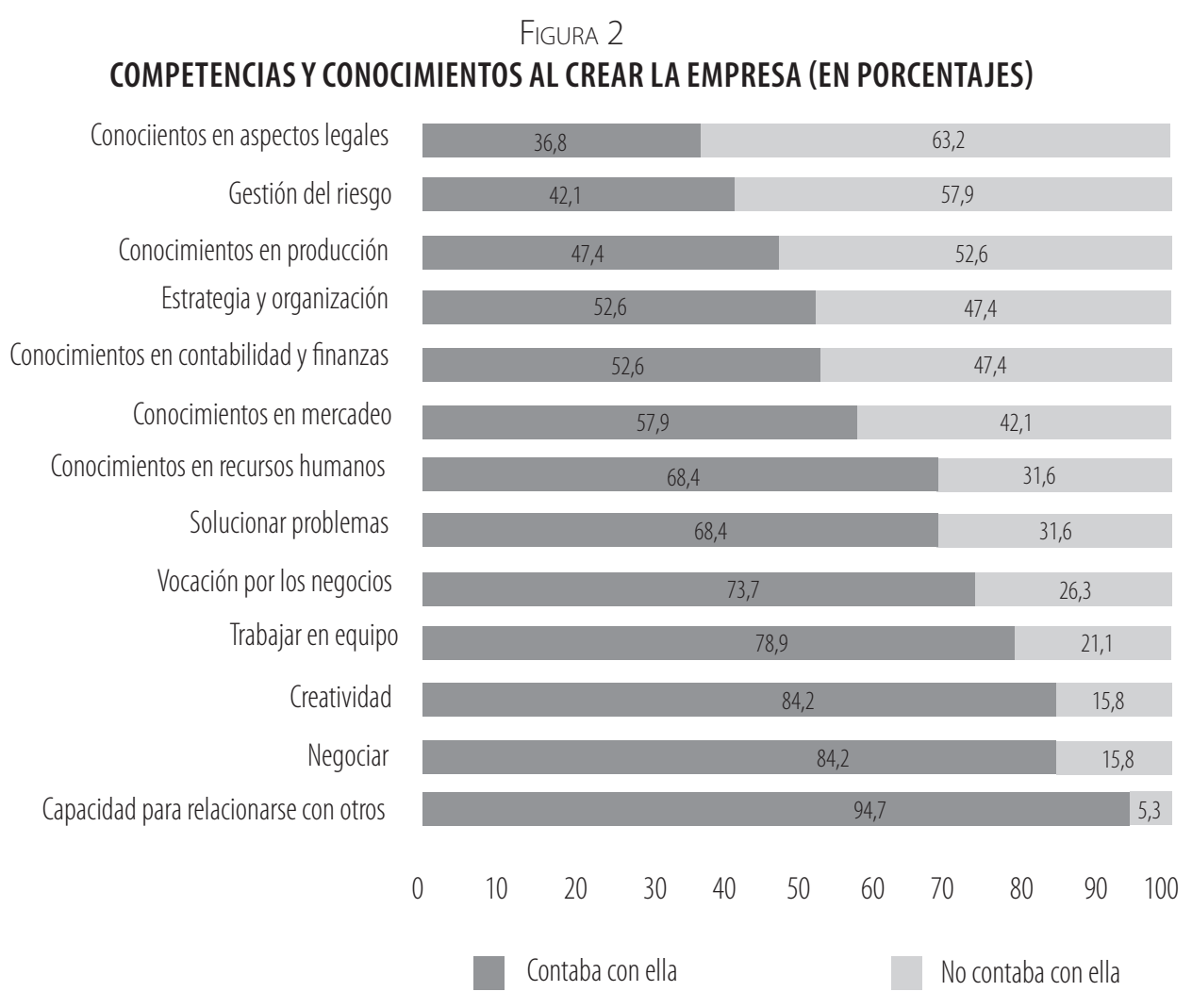

Fuente: Elaboración propia.

En lo concerniente al lugar de adquisición de los conocimientos y competencias, se muestra un complemento del contexto familiar y social, la experiencia laboral y la formación universitaria (Tabla 8). 
TABLA 8

LUGARES DONDE ADQUIRIÓ LAS COMPETENCIAS Y CONOCIMIENTOS CON QUE CONTABA AL CREAR LA EMPRESA

\begin{tabular}{|c|c|c|c|c|c|}
\hline \multirow[b]{2}{*}{ CONOCIMIENTO O COMPETENCIA } & \multicolumn{5}{|c|}{ DÓNDE LAS ADQUIRIDO } \\
\hline & UNIVERSIDAD & OTROS ESTUDIOS & EXPERIENCIA LABORAL & $\begin{array}{l}\text { CONTEXTO FAMI- } \\
\text { LIAR Y SOCIAL }\end{array}$ & TOTAL \\
\hline Vocación por los negocios & 14,3 & 14,3 & 28,6 & 42,9 & 100,0 \\
\hline Solucionar problemas & 14,3 & 14,3 & 28,6 & 42,9 & 100,0 \\
\hline Capacidad para relacionarse con otros & 11,1 & 5,6 & 55,6 & 27,8 & 100,0 \\
\hline Gestión del riesgo & 37,5 & 12,5 & 25,0 & 25,0 & 100,0 \\
\hline Negociar & 13,3 & 0,0 & 40,0 & 46,7 & 100,0 \\
\hline Trabajar en equipo & 18,8 & 12,5 & 43,8 & 25,0 & 100,0 \\
\hline Creatividad & 18,8 & 0,0 & 31,3 & 50,0 & 100,0 \\
\hline Aspectos legales & 50,0 & 12,5 & 25,0 & 12,5 & 100,0 \\
\hline Contabilidad y finanzas & 72,7 & 0,0 & 18,2 & 9,1 & 100,0 \\
\hline Producción & 60,0 & 0,0 & 40,0 & 0,0 & 100,0 \\
\hline Mercadeo & 66,7 & 0,0 & 33,3 & 0,0 & 100,0 \\
\hline Recursos humanos & 50,0 & 14,3 & 28,6 & 7,1 & 100,0 \\
\hline Estrategia y organización & 54,5 & 0,0 & 36,4 & 9,1 & 100,0 \\
\hline
\end{tabular}

Fuente: Elaboración propia.

En lo referente a la experiencia en empresas faIlidas, una mayoría no intentó en el pasado crear otras empresas (74\%). Del 26\% que intentaron crear empresas anteriormente a la empresa actual, manifestaron como las principales razones, por las que no se cristalizaron esos intentos, la falta de experiencia del emprendedor y las condiciones del contexto en que se originaba la empresa, tales como las condiciones de mercado y de financiamiento.

Con respecto a la asociatividad, lo más frecuente fue el inicio de sus empresas individualmente (58\%), aunque también fue importante los equipos de dos o más personas (42\%). De estos últimos, la mitad (21\%) buscaron equipos de personas con habilidades complementarias que desempeñaran diferentes roles para la empresa, y la otra mitad personas con habilidades semejantes, similares disciplinas y experiencias laborales.

Los datos evidencian poca interrelación gremial de los entrevistados, solo un 11\% pertenecen a un grupo empresarial.

\section{El mercado y el contexto}

En cuanto a las condiciones de mercado y de contexto que afectaron la empresa en su creación, se presentan en este apartado los resultados sobre la información para la toma de decisiones respecto al emprendimiento y las dificultades y apoyos que tuvieron las personas entrevistadas en sus procesos de creación de empresas.

En relación con la información y fuentes sobre oportunidades de negocios, la interrelación con familiares, amigos o conocidos, compañeros de estudio y de trabajo fue la principal fuente de in- 
formación sobre el negocio (44,4\%). Otra fuente de información importante fue la experiencia en trabajos previos (40,7\%). La Universidad no constituye un espacio importante para la generación de ideas de negocios (7,4\%), tampoco los medios de comunicación colectiva $(7,4 \%)$.

En lo que respecta a la disposición de información básica para decidir sobre la creación de la empresa, alrededor de un 32\% contó con un plan de negocios, un 26\% evaluó el costo de oportunidad de emprender en comparación con el seguimiento en la actividad laboral que realizaban en ese momento, y solo un 5\% realizó cálculos del Valor Actual Neto (VAN) y Tasa Interna de Retorno (TIR) para evaluar la rentabilidad del proyecto de inversión.

En lo que respecta a las dificultades para la creación y puesta en marcha de la empresa, la inversión financiera necesaria y el conocimiento pertinente fueron las principales limitantes (Tabla 9).

\section{TABLA 9 \\ DIFICULTADES PARA LA PUESTA EN MARCHA DE LA EMPRESA}

\begin{tabular}{|c|c|c|}
\hline DIFICULTADES & MENCIONES & PORCENTAJE \\
\hline Inversión necesaria para ser competitivo & 10 & 52,6 \\
\hline $\begin{array}{l}\text { Conocimiento y experiencia en el } \\
\text { ámbito del negocio }\end{array}$ & 8 & 42,1 \\
\hline $\begin{array}{l}\text { Conocimientos sobre administración de } \\
\text { empresas }\end{array}$ & 5 & 26,3 \\
\hline Dinamismo del mercado & 4 & 21,1 \\
\hline Situación económica del país & 4 & 21,1 \\
\hline Red de contactos personales & 2 & 10,5 \\
\hline Disponibilidad de materia prima & 2 & 10,5 \\
\hline Tamaño del marcado local & 2 & 10,5 \\
\hline Otras & 4 & 21,0 \\
\hline
\end{tabular}

Nota. Se podía realizar más de una mención, el porcentaje se calcula respecto a 19.

Fuente: Elaboración propia.
En los primeros años de contar con la empresa, más del 40\% experimentó problemas en conseguir clientes y proveedores adecuados (Tabla 10).

TABLA 10

\section{PROBLEMAS ENFRENTADOS EN LOS PRIMEROS AÑOS DE LA EMPRESA}

\begin{tabular}{l|c|c}
\hline \multicolumn{1}{c|}{ PROBLEMAS } & MENCIONES & PORCENTAJE \\
\hline Obtener clientes & 9 & 47,4 \\
Conseguir proveedores adecuados & 8 & 42,1 \\
Contratar personal adecuado & 5 & 26,3 \\
Financiar y manejar el flujo de caja & 5 & 26,3 \\
Adquirir el equipamiento adecuado & 5 & 26,3 \\
Adaptar el producto/servicio a las & 5 & 26,3 \\
necesidades del mercado & 2 & 10,5 \\
Manejar las relaciones con los clientes & 2 & 10,5 \\
Certificar estándares de calidad & 1 & 5,3 \\
\hline Manejar las operaciones de la empresa & & \\
\hline
\end{tabular}

Nota. Se podía realizar más de una mención, el porcentaje se calcula respecto a 19

Fuente: Elaboración propia.

Con respecto a los financiamientos, la fuente principal de fondos para la creación de las empresas fue los ahorros de los fundadores y los que suplen personas allegadas a éstos, tales como familiares y amigos (Tabla 8). 
TABLA 11

\section{FUENTES DE FONDOS PARA EL INICIO DE LA EMPRESA}

\begin{tabular}{|l|c|c|}
\hline \multicolumn{1}{|c|}{ FUENTES } & \multicolumn{2}{c|}{ PORCENTAJES } \\
\hline Personal y familiar & 65,2 & \\
\hline Ahorros personales de los fundadores & & 52,2 \\
\hline Parientes y amigos & & 13,0 \\
\hline Entes financieros & 26,1 & \\
\hline Crédito personal (fundadores) & & 8,7 \\
\hline \multicolumn{1}{|c|}{ Préstamos de bancos a la empresa } & & 8,7 \\
\hline Préstamos de otras instituciones & & 8,7 \\
\hline Subsidiarios & 8,7 & \\
\hline Subsidios Instituciones públicas nacionales & & 4,3 \\
\hline Empresa donde anteriormente trabajaba & & 4,3 \\
\hline Total & 100,0 & 100,0 \\
\hline
\end{tabular}

Fuente: Elaboración propia.
El poco acceso a recursos financieros externos tuvo como consecuencia la reducción de la escala del proyecto, el crecimiento de la empresa en una tasa menor a la deseada y la dedicación del emprendedor/a a otros proyectos adicionales a la empresa que permitieran adquirir liquidez y capital.

Sobre la temática de apoyos recibidos y requeridos por las personas entrevistadas al crear la empresa y en sus primeros años, la mayoría $(45,5 \%)$ provinieron de la familia y los amigos. Una parte de quienes fueron entrevistados obtuvieron apoyo de las universidades en la creación de su empresas (15,8\%), específicamente en cursos de capacitación y apoyo logístico. Otros apoyos provinieron de instituciones públicas, cámaras empresariales, entre otros (38,7\%).

Los requerimientos planteados por las personas entrevistadas para las universidades, en relación con el apoyo en la etapa inicial y en el desarrollo de las empresas se presentan en la Figura 3.

FIGURA 3

\section{APOYO DE LAS UNIVERSIDADES REOUERIDO POR LAS EMPRESAS}
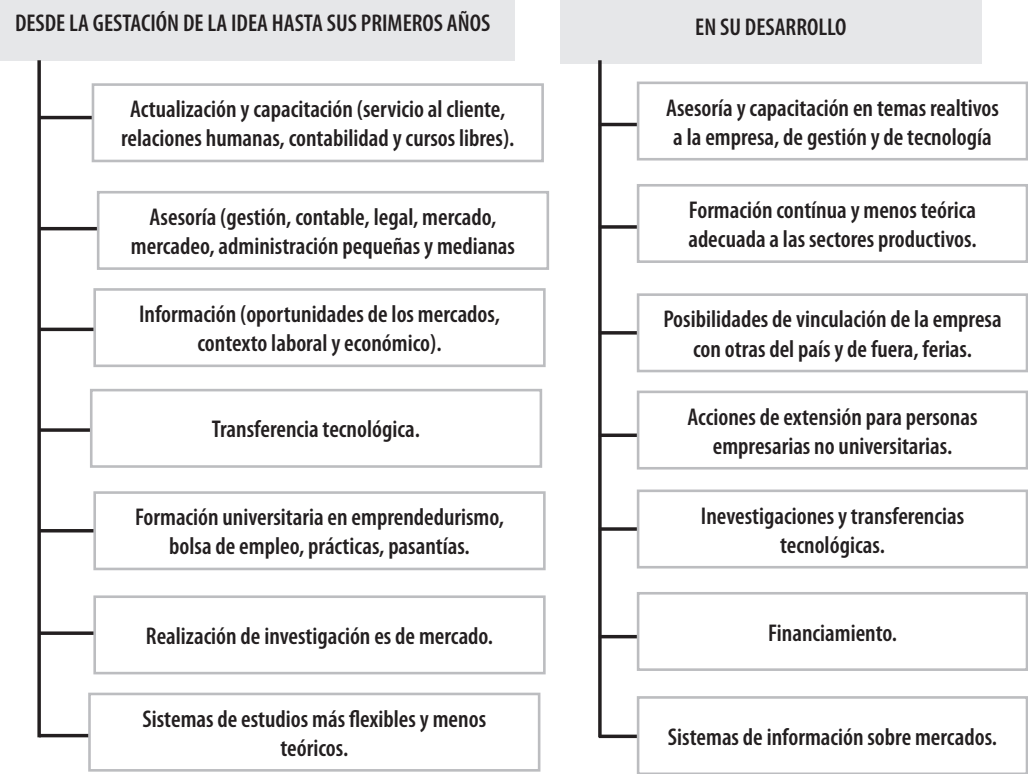

Fuente: Elaboración propia. 


\section{CONCLUSIÓN}

Las empresas estudiadas son de tamaño muy pequeño, ubicadas principalmente en la zona central, en la cual se concentra la mayor actividad económica del país. Este hecho está relacionado con las actividades comerciales de ámbito muy reducido que desarrollan, en las que la mano de obra necesaria para atenderlos es de tipo familiar.

El emprendedurismo femenino fue más fuerte en el periodo de estudio pues proporcionalmente, una mayor cantidad de mujeres se hicieron empresarias. Priman las motivaciones de lograr desarrollo personal, por encima, incluso, de las de obtener mayores ingresos. Una razón de esta situación, puede estar relacionada con que los salarios de nivel profesional, que recibe una persona graduada, no variarían mucho de los ingresos por ser micro o pequeño empresariado, por lo que la mera rentabilidad económica no es suficiente para explicar el emprendedurismo.

En general, hay una mínima proporción de entrevistados que en su niñez tuvieron padres o madres empresarios. El autoempleo sí presenta un porcentaje importante en el caso de los padres, por tanto este rol puede haber influido en que estas personas decidieran crear empresas propias. La mayoría de las madres realizaban trabajo no remunerado en las tareas del hogar y la familia, en correspondencia con la norma social del rol de la mujer hace 30 o más años en que las personas entrevistadas estaban en su niñez. La experiencia laboral con que contaban las personas al momento de emprender pudo haber compensado la débil figura de padre o madre empresaria.

Los estudios internacionales muestran que tiene mayores posibilidades de supervivencia un emprendimiento que es llevado a cabo por un equipo de socios con formaciones y capacidades complementarias, que uno conducido por un solo emprendedor (Kantis, Ishida \& Komori, 2002). Esto debe promoverse en los emprendimientos del estudiantado de la UNED.

El tema de asociatividad gremial no se profundiza en esta investigación, pero la poca participación en algún grupo empresarial, puede dar indicios sobre el grado de madurez de la empresa en términos de sus relaciones externas con sus iguales.

La Universidad no constituyó un espacio importante para la generación de ideas de negocios, aunque en la actualidad se puede observar mayores acciones para apoyar el emprendedurismo en la UNED.

Debido al riesgo que presentan los emprendimientos en sus primeros años, en los que hay una alta tasa de mortalidad empresarial, este tipo de empresas tiene como fuente principal de fondos para su creación, los provenientes de los ahorros de los fundadores y los que suplen personas allegadas a estos, tales como familiares y amigos. Aunque en la actualidad, debido a la Ley para el Fortalecimiento de las Pequeñas y Medianas Empresas, los bancos públicos tienen líneas de crédito con condiciones preferenciales para micro, pequeña y mediana empresa, es natural que las personas emprendedoras, debido al riesgo de su actividad, prefieran no apalancar la empresa hasta que ésta tenga un mayor desarrollo, aunque esto implique que no dispongan de capital financiero suficiente.

Por último, para que estas microempresas puedan crecer requieren de una efectiva red de apoyo. En este sentido, las universidades pueden representan un elemento fundamental por medio de los programas de emprendedurismo. 


\section{REFERENCIAS}

CEPAL. (1999). La PYME en Centroamérica y su vinculación con el sector externo. México: CEPAL.

Fundación Arias para la Paz y el Progreso Humano. (2006). Directorio de organizaciones para la promoción de la micro, pequeña y mediana empresa en Centroamérica. San José, Costa Rica.

Hernández, R. (2001). Elementos de competitividad sistémica de las pequeñas y medianas empresas (PYME) del istmo centroamericano. México: CEPAL.

Kantis, H. (2004). Desarrollo emprendedor: América Latina y la experiencia internacional. Washington, DC: Banco Interamericano de Desarrollo.

Kantis, H., Ishida, M. \& Komori, M. (2002). Empresarialidad en economías emergentes. Creación de empresas en América Latina y el este de Asia. Washington, DC: Banco Interamericano de Desarrollo.

MEIC. (2009). PYME Costa Rica. Información Estadística. Recuperado el 30 de octubre del 2009 de: http://www. pyme.go.cr/svs/informacion_estadistica/default.aspx

Postigo, S. Tamborín, M.F. (2004, marzo). Educación y Entrepreneurship en Argentina: El caso de la Universidad de San Andrés, Argentina. Trabajo presentado en el Congreso de la Red Motiva, Valencia, España. Recuperado el 18 enero del 2007 de: http://www.uv.es/motiva/ Congval.htm

Procomer (2008). Análisis de las Estadísticas de Exportación, 2007. San José, Costa Rica.

Ribeiro D. (2004, marzo). Análisis aproximativo de las variables influyentes en el empresario y emprendedor de éxito en el contexto teórico internacional. Trabajo presentado en el Congreso de la Red Motiva, Valencia, España. Recuperado el 18 enero del 2007 de: http://www.uv.es/ motiva/CongVal.htm

Ulate, A. (2001). El rol de las MIPYMES en Costa Rica ¿Generar empleo o acumular valor? San José, Costa Rica: Instituto de Investigaciones en Ciencias Económicas, Universidad de Costa Rica.

Zevallos, E. (2003). Micro, pequeñas y medianas empresas en América Latina. Revista de la Cepal, 79, pp. 53-70.

Recibido: 04-11-2009

Aceptado: 22-06-2010 